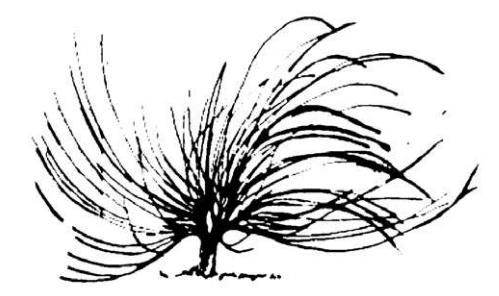

\title{
Influencia de las tecnologías celulares en el rendimiento académico
}

\author{
Giselle León León ${ }^{l}$ \\ Universidad Nacional de Costa Rica \\ Heredia, Costa Rica \\ leongiselle@hotmail.com \\ Luis Bent Solano 3 \\ Universidad Nacional de Costa Rica \\ Heredia, Costa Rica \\ alfredo-_92@hotmail.com
}

\author{
Deyber Alvarado Fernández $z^{2}$ \\ Universidad Nacional de Costa Rica \\ Heredia, Costa Rica \\ deyjoalvafer@gmail.com \\ Hellen Navarro Jiménez ${ }^{4}$ \\ Universidad Nacional de Costa Rica \\ Heredia, Costa Rica \\ hellnava@gmail.com
}

\section{Resumen}

El presente artículo surge a partir de una investigación sobre la influencia de tecnologías celulares en el rendimiento académico, la cual se realizó bajo el enfoque mixto en la clasificación de mixto dominante, en cuanto a instrumentos para recopilar la información, se aplicó un cuestionario a dieciséis estudiantes de sétimo año, se realizaron observaciones al desarrollo de las lecciones y se entrevistó al docente de enseñanza de las ciencias de un Liceo del Área Metropolitana costarricense. Algunos de los aportes fueron resaltados en matrices y contrastados mediante la triangulación de informantes y teoría. Dentro de las principales conclusiones se indican, que los

Recibido: 29 de abril de 2013 - Aprobado: 11 de octubre de 2013

1 Académica de la División de Educología, Universidad Nacional de Costa Rica. Máster en Administración Educativa, Bachiller en la Enseñanza de las Ciencias y Licenciada en Ciencias de la Educación con Énfasis en Didáctica de Ciencias. Estudiante del Doctorado en Educación de la Universidad Autónoma de Durango, México.

2 Estudiante de Enseñanza de las Ciencias, Universidad Nacional.

3 Estudiante de Enseñanza de las Ciencias, Universidad Nacional.

4 Estudiante de Enseñanza de las Ciencias, Universidad Nacional. 
celulares son los dispositivos electrónicos que se encuentran mayoritariamente presentes en las clases, repercutiendo en el rendimiento de los estudiantes de la muestra.

Palabras claves: rendimiento, celulares, influencia, sétimo, ciencias.

\begin{abstract}
This article emerges from a research about the influence of cell phones technologies on high school students' performance. This research was conducted under the mixed approach in the classification of dominant mixed. Regarding the instruments for gathering the information, a questionnaire was applied to sixteen students from seventh grade, observations of lessons were made, and an interview to the Science teacher of a High School in the San Jose central area was held. Some of the contributions were highlighted in matrixes and compared through informers' triangulation and the theory. Among the main findings, they indicate that cell phones are electronic devices that are commonly present in these Science classes, affecting the students' performance of the sample.
\end{abstract}

Keywords: performance, cellular, influence, seventh, science.

\title{
Introducción
}

$\mathrm{E}$ $\mathrm{s}$ un hecho, que las tecnologías de la información y la comunicación (TIC), están generando, una transformación en las relaciones culturales entre las personas: cambios en las formas de actuar, pensar, sentir, comunicarse, enseñar y aprender.

Como consecuencia, en el campo educativo emerge una serie de inquietudes relacionadas con el uso de estos recursos en los procesos escolares. Una de esas inquietudes es cómo el uso continuo de estos recursos en las aulas de clase podría afectar el rendimiento académico de los estudiantes.

Lo anterior ya que en la actualidad, la mayoría de estudiantes cuentan con tecnologías tales como teléfonos celulares, ipods, reproductores, mp3, cámaras, computadoras entre otros, los cuales llevan a 
los centros de formación y pueden representar un elemento de distracción si no se les da un adecuado manejo.

Ante esa realidad, más que atacar el problema enviando boletas o reprimiendo verbalmente a los estudiantes, el profesor debe ser ágil y capaz de adecuarse a las necesidades de los estudiantes. Por lo tanto, una alternativa es incorporar dichas tecnologías en el proceso de mediación, de manera que éstas faciliten el proceso de aprendizaje ya que en términos generales las TIC incrementan el logro de los objetivos de aprendizaje por los cambios que promueve en la mediación pedagógica, por la diversidad de experiencias novedosas que ofrecen, por la posibilidad que brinda para que el estudiantado avance de acuerdo con sus necesidades e intereses.

Ante el contexto descrito, la finalidad de este artículo consiste en contribuir al debate sobre la influencia en el rendimiento académico que genera el uso de tecnologías como el celular en el aula, a la luz del estudio realizado en una sección de sétimo nivel de la secundaria costarricense.

\section{Antecedentes}

Se han llevado a cabo varios estudios a nivel nacional acerca de las TIC en el proceso educativo, uno de ellos es el propuesto por Cuevas y Alvarez en el año 2010, cuyo objetivo fue analizar de manera comparativa las condiciones de tenencia y acceso de las principales tecnologías infocomunicacionales y el uso que hacen de ellas los estudiantes y docentes. S e realizó en 49 colegios de secundaria de Costa Rica con cobertura nacional. El estudio permitió determinar que los estudiantes tienen relación con las tecnologías a pesar de las brechas socioeconómicas y de la zona de residencia, la gran mayoría las conoce y las usa en igual condición se encuentran los docentes. Sin embargo, la utilización de tales herramientas como medios didácticos es todavía una expectativa, ya que utilizan pizarra y marcador para impartir sus clases.

Por otro lado, se puede indicar la investigación de Leitón (2010), cuyo objetivo fue analizar los factores afectivos y cognitivos que pueden incidir en el rendimiento académico, en la asignatura de Matemática, en la modalidad de Nocturno Académico: estudio de caso en la sección 9-3 del Colegio Nocturno de Guácimo, durante el año 2008. Dentro de los principales hallazgos se encontraron que en el proceso de aprendizaje de la matemática influyen factores afectivos como: relación con los padres, 
triple rol trabajo, estudio y familia y poco tiempo para repasar; a nivel cognitivo se enumeraron falta de hábitos de estudio, la propia dificultad de la materia, la actitud frente a la materia, los docentes y la falta de apoyo familiar, lo anterior genera apatía por la materia, el desinterés, la desmotivación, ausentismo y el bajo rendimiento académico.

De igual manera se puede citar el aporte de González, Leal y Romero (2011) sobre los aspectos que más afectaron el desempeño escolar de una muestra de estudiantes de Guararí en su rendimiento académico. Las principales conclusiones fueron la relación afectiva y la colaboración de los padres con sus hijos es fundamental para que logren el éxito escolar, el nivel educativo de los padres no es determinante, pero entre mayor escolaridad tienen, mayor es el rendimiento académico de sus hijos.

Por último, se puede indicar el estudio realizado por Matarrita, Serrano, Torres, y León en el 2012 sobre el uso de recursos tecnológicos para la implementación del tema transversal cultural ambiental para el desarrollo sostenible haciendo énfasis en problemáticas ambientales relacionadas con los contenidos del programa de ciencias de séptimo año de la Educación Básica. Dentro de los principales resultados se destaca que los estudiantes exponen su gran interés por recibir las lecciones utilizando recursos tecnológicos, por otro lado se tiene la opinión no muy favorable de algunos docentes, quienes no muestran mucho interés por el tema, además de no estar capacitados en su uso. Tal como se muestra, no se lograron identificar investigaciones que abordaran el uso del teléfono en clase y su posible relación con el rendimiento académico.

\section{Problema}

¿Influye el uso de tecnologías en el aula en el rendimiento académico de estudiantes de secundaria?

\section{Objetivos}

Objetivo general: Analizar la influencia del uso de tecnologías celulares por parte de los estudiantes de una sección de sétimo de un colegio de San José, en el rendimiento académico de las lecciones de Ciencias. 


\section{Objetivos específicos:}

- Identificar los tipos de tecnologías que disponen los estudiantes y usan en el desarrollo de las lecciones de Ciencias.

- Determinar la forma en que se usan las tecnologías celulares por parte de los estudiantes en las clases.

- Identificar la manera en la que influye la presencia de estas tecnologías en el rendimiento académico de los estudiantes.

- Describir las características de la mediación pedagógica desarrollada por el docente en las clases de ciencias.

\section{Marco Teórico}

\section{La tecnología presente en la educativa}

Los individuos necesitan comunicarse y para ello se crean modernos y costosos instrumentos tecnológicos que muchas veces no están al alcance de todas las personas, pero aun así, los adquieren. Las implicaciones que genera la adquisición de nuevas tecnologías pueden traer consecuencias positivas y negativas; según Piscitelli, (s.f.) "(...) los chicos que hoy tienen entre 5 y 15 años son la primera generación mundial que ha crecido inmersa en estas nuevas tecnologías, han pasado toda su vida rodeados de computadoras, video games y el resto de los gadgets digitales (...)" (p. 2 ), por lo que no es de extrañar que dichas tecnologías estén presentes en su diario vivir, por tanto, también están presentes en las instituciones educativas.

Las tecnologías repercuten en la educación de las nuevas generaciones, además el sistema educativo en el que se desarrollan esas nuevas generaciones van quedando obsoleto y no se ajusta a los cambios que viven las sociedades, por ende, “(...) los estudiantes de hoy (los milenaristas) han cambiado de forma radical, y no son los sujetos para los cuales el sistema educativo fue diseñado durante siglos (...)"( Piscitelli, s.f., p. 2).

Por su parte, Piscitelli (s.f.) señala que emergen 2 tipos de grupos: los nativos digitales y los inmigrantes digitales, en donde los nativos corresponden a las nuevas generaciones nacidas en un mundo tecnológico identificados por los estudiantes y los inmigrantes digitales son las generaciones pasadas, en las cuales la tecnología no generó una gran significancia, donde se identifican a muchos de los actuales docentes. 
Por consiguiente, actualmente los docentes "obsoletos" enseñan a estudiantes "avanzados" tecnológicamente hablando, lo cual genera un enorme desfase en el campo educativo.

\section{Presencia de las tecnologías en el aula}

Los estudiantes actuales viven en un mundo tecnológico por lo que no es lo mejor prohibir o "eliminar" las tecnologías de las aulas porque eso sería algo utópico, son los docentes los llamados a mediar el uso de ellos con el objetivo de enriquecer el proceso educativo. Al respecto Reyes en el 2008 indica: “(...) las computadoras en las casas no siempre tienen usos educativos positivos, en realidad, estas rara vez están conectadas con la escuela"(p.4 ), pero esa situación no es del todo culpa de los estudiantes, es también falta de interés de los docentes en tratar de vincular las tareas educativas con las tecnologías que los estudiantes posean.

En Costa Rica más de la mitad de la población estudiantil posee algún aparato electrónico (en su mayoría teléfono celular), debido al acceso que tienen las personas para adquirirlos hoy. Además, cuentan con una gama de opciones para adquirir el aparato que desee. Ante esta situación es común observar a estudiantes con aparatos celulares, lo anterior lo afirma Sánchez (2011).

(...) los celulares por su bajo costo están al alcance de la población, su amigabilidad hace que su uso sea accesible y el avance tecnológico ha logrado que no solo sea un dispositivo de comunicación verbal, sino de envío de mensajes escritos, grabación de voz, videos, agenda, juegos que no requiere de una conexión especial, otra característica que lo distingue, es su facilidad de traslado debido a su pequeño tamaño y de ahí que algunos investigadores lo consideren como el dispositivo que permite el acceso del conocimiento en la palma de la mano o como lo ha anunciado la UOC: la universidad en el bolsillo (...) (p.198)

La diferencia de la educación de "ayer" a la educación de "hoy" se puede resumir según Reyes (2008) de la siguiente manera:

En el siglo pasado y principios de este, la escuela era un ambiente privilegiado que concentraba el conocimiento dedicado a la 
educación del niño y del adolescente. Pero, además, la escuela concentraba no sólo el conocimiento, sino también las "herramientas pedagógicas", es decir, la tecnología imprescindible para impartir la enseñanza: pupitres, libros, lápices, plumas, tinteros, tinta, cuadernos, pizarrones, tizas, mapas. En las casas estos instrumentos eran escasos o inexistentes." (p.4)

Con lo anterior, se explicita que así como las sociedades avanzan, las tecnologías también en el mejor de los casos, la educación debería de avanzar con esos 2 elementos, sin embargo, hoy los estudiantes poseen muchos más recursos que los que poseen las instituciones.

\section{La tecnología como recurso didáctico}

La tecnología educativa se postula como uno de los campos en los cuales el educador puede hacer uso, como recurso didáctico para el desarrollo de procesos activos en el aprendizaje, pero de antemano la mayor preocupación es el papel que debe cumplir el docente en la mediación utilizando tecnología educativa Galvis, (2004). En ese mismo sentido Olier (s.f.), explica, el uso de las tecnologías en un salón de clases no sirve de nada si el docente no pretende darle el uso pedagógico que se merece, de esto depende la estrategia pedagógica; que pase de ser un proceso que esté sólo centrado en la actividad del docente a uno que gire alrededor del educando, sin estar diciendo que desaparecerá el docente; sino que servirá de apoyo para crear ambientes educativos agradables y ricos en oportunidades para llegar al conocimiento a partir de la experiencia.

Con el fin de garantizar un entorno satisfactorio del aprendizaje de los estudiantes a través de la tecnología educativa, el docente podría usar un sistema de trabajo en el cual el educando sea capaz de llegar a construir su propio conocimiento a partir de la guía del profesor, dejando de antemano la idea de ser partícipes de una tendencia algorítmica en la tecnología educativa, es decir “ (...) una enseñanza del tipo tubería" (Galvis, 1991, p.19).

En ese sentido, se podría pretender que el estudiante a partir del proceso de enseñanza y aprendizaje, sea partícipe de la construcción de su propio conocimiento, utilizando estrategias y materiales propios de la tecnología educativa. 


\section{Mediación pedagógica}

Es un proceso de gran relevancia en la educación, se basa en saber utilizar los contenidos y la forma de tratar diferentes temas con el fin de hacer del proceso de enseñanza y aprendizaje un momento de gran interés para el educando, puede opinar y expresar sus experiencias, enriqueciendo la clase, además donde pueda utilizar su creatividad para hacer más agradable este proceso.

Al respecto Gutiérrez y Prieto (2004), conceptúan medicación pedagógica como "(...) el tratamiento de contenidos y de las formas de expresión de los diferentes temas a fin de hacer posible el acto educativo, dentro del horizonte de una educación concebida como participación, creatividad, expresividad y relacionalidad " (p. 50).

De igual manera, Pérez (2009) menciona la mediación pedagógica parte de la necesidad de promover experiencias de aprendizaje, de lograr la interactividad del estudiante de manera integral como ser humano, comprender que el acto pedagógico no puede ser directivo, es una forma de interacción entre personas con niveles de experiencias diferentes, donde se parte de la necesidad de compartir para construir en equipo, en un determinado contexto.

En la mediación pedagógica, lo que importa es el sentimiento que tenga el estudiante hacia la clase, es necesario realizar actividades, ejercicios y procedimientos placenteros, significativos, novedosos y requeridos para el bienestar de los educandos, tal como lo indica Malagón 2009, citando a Gutiérrez y Prieto 1999 “(...) la mediación puede surgir del trabajo en el aula y depende casi siempre de la capacidad y la pasión del docente" (s.p.).

En relación con lo anterior, Gutiérrez y Prado (2004) afirman que en la mediación pedagógica no hay prisa, dado que no interesa tanto la acumulación de la información y los productos programados en cuanto a los procesos que se abren a la reflexión, inherente a lo imprevisible. Es decir, en la mediación pedagógica los ritmos de aprendizaje de cada individuo son respetados, el docente no está dirigido a transmitir una serie de contenidos, sino que, se orienta a que el educando logre reflexionar acerca de lo que hace o podría hacer con el objeto de aprendizaje. En complemento, Bessone (2005) considera que cuando se hace referencia a la mediación pedagógica se debe tomar en cuenta que implica reconocer el potencial de aprendizaje social y experiencial ínsito en la mediación y consecuentemente aprovechar 
las incontables oportunidades que el aula y la escuela nos dan para introducir una mirada distinta del conflicto, sacándolo de la categoría de aquello que hay que acallar, para poder pensado como oportunidad de crecimiento personal y colectivo (s.p.).

Es decir, la acción social es inherente del proceso de enseñanza y aprendizaje mediatizado, por eso, el educador debe considerar situaciones de aprendizaje en las que los educandos puedan compartir, comparar sus ideas con otros y la acción mental que se produzca de la relación que se establezca entre pares será sinónimo de aprendizaje y desarrollo. El aprendizaje que espera provocar la mediación pedagógica es un aprendizaje para la vida, es decir, que pueda ser aprovechado por el alumno cuando lo necesite.

Entonces, la mediación pedagógica está caracterizada por la relación dinámica del estudiante, con sus pares, el docente y el medio social que le rodea, donde el educador es quien orienta intencionalmente la actividad, para que el joven construya aprendizajes que le permitan autoorganizar sus ideas con el fin de que le sean útiles en su cotidianidad al enfrentar nuevas experiencias.

\section{Marco Metodológico}

\section{Paradigma}

Esta investigación se llevó a cabo bajo un paradigma naturalista, también llamado naturalista-humanista o interpretativo, el cual “(...) se centra en el estudio de los significados de las acciones humanas y de la vida social" (Barrantes, 2006, p. 60). En este caso, el proceso educativo, a la luz de las tecnologías en un grupo de sétimo nivel.

\section{Enfoque de estudio}

La presente investigación se ubica dentro del enfoque mixto, a su vez responde a la clasificación de enfoque mixto dominante, sustentado por Hernández, Fernández y Baptista (2006) “(...) el estudio se desarrolla bajo la perspectiva de alguno de los dos enfoques, la cual prevalece, y la investigación mantiene un componente del otro enfoque" (p. 773). En la presente investigación dominó el enfoque cualitativo con algunos elementos de corte cuantitativo, como el cuestionario, el cual generalmente se asocia a investigaciones cuantitativas. 


\section{Tipo de estudio}

El tipo de estudio en el cual se enfocó está investigación fue fenomenológica, ya que se describen los fenómenos, es decir, se explica la esencia de las experiencias de los actores, en este caso el rendimiento académico de los estudiantes y su relación con el uso de tecnologías en el aula (Gurdián, 2007).

\section{Sujetos participantes}

Los individuos que participaron en el estudio fueron un docente de Ciencias, quien es egresado de una universidad privada, contaba con dieciocho años de experiencia en el momento del estudio. De igual manera, se contó con el aporte de un grupo de estudiantes de sétimo año conformado por 10 hombres y 5 mujeres ${ }^{5}$, que se encontraban cursando el tercer ciclo en un liceo del área metropolitana.

\section{Instrumentos y técnicas utilizadas}

Para recopilar la información se aplicó un cuestionario dirigido a los estudiantes, una entrevista al docente y una observación de clase.

\section{- Cuestionario}

El cuestionario fue aplicado a los estudiantes de la sección 7-A, el cual estaba constituido por 11 preguntas semi-estructuradas. El objetivo fue conocer los tipos de tecnologías que poseían y usualmente utilizan en clase, así como su opinión en cuanto a la relación con el rendimiento académico.

\section{- $\quad$ Entrevista}

La entrevista se dirigió al docente de la clase de sétimo, la cual se realizó en uno de los recesos, contenía 16 preguntas abiertas con el propósito de conocer la opinión del docente con respecto al uso, regulación e implementación de tecnologías (aparatos electrónicos) en el quehacer educativo, la mediación pedagógica específicamente el tipo de estrategias o técnicas que utiliza para desarrollar los temas en clase y su opinión sobre el interés de los estudiantes en la materia (rendimiento).

5 La investigación se realizó en el II ciclo del año 2012, como parte de las actividades del curso Innovación y Producción Educativa. 


\section{- Observación}

La observación se realizó durante el desarrollo de 4 lecciones de Ciencias, tenía como propósito fundamental contrastar la opinión de los estudiantes con lo indicado por el docente y visualizar la mediación pedagógica, la cual se recopiló mediante un registro anecdótico.

\section{Consideraciones éticas}

La investigación se llevó a cabo en un liceo de San José, al cual se pudo ingresar gracias a un documento dirigido al director, en el que se solicitaba la colaboración para realizar la investigación.Tanto los instrumentos como sus ítems fueron realizados por los investigadores y se derivaron de los objetivos de la investigación.

\section{Análisis de resultados}

En este apartado se muestran los resultados obtenidos mediante la aplicación de los tres instrumentos. Algunos de los resultados son presentados mediante matrices, los cuales se muestran de acuerdo con el tipo de instrumento.

A partir del cuestionario se pudo identificar que de los dieciséis estudiantes encuestados sólo uno no contaba con celular, lo que evidenció que los estudiantes tienen gran acceso a la telefonía celular tal como se muestra en la matriz 1. Lo anterior es afirmado a nivel teórico al indicar que en Costa Rica más de la mitad de la población estudiantil posee algún aparato electrónico (en su mayoría teléfono celular), debido al acceso que tienen las personas para adquirirlos hoy por (...) su bajo costo están al alcance de la población (...) (Sánchez, 2011, p. 198), por lo tanto, el manejo dentro del aula, debe empezar a preocupar a los docentes ya que es un hecho que estos aparatos están presentes en los centros de aprendizaje. Lo indicado antes se pudo constatar con la observación, puesto que los estudiantes poseían y utilizaban libremente los aparatos de comunicación en la clase de Ciencias. 
Tabla 1. Tipos de aparatos electrónicos que tienen y usan en clase los estudiantes

\begin{tabular}{|c|l|}
\hline Estudiante & \multicolumn{1}{|c|}{ Tipo aparato electrónico } \\
\hline A B,C,D & Celular Samsung Galaxy \\
F & Celular Nokia \\
Celular Nokia, Ipod Apple \\
G & Celular LG \\
H & Celular Sony Ericsson \\
I & Celular LG \\
J & Celular Sony Ericsson mini, PsP gol \\
K & Celular Nokia \\
L & Celular Alcatel \\
M & Celular Samsung \\
N & Celular Sony Ericsson x10 \\
O & Celular Nokia C2 \\
\hline
\end{tabular}

Fuente: Resultados obtenidos del cuestionario aplicado a los estudiantes de la sección 7-A. 
Tabla 2. Motivos por el cual los estudiantes utilizan su teléfono celular en el aula

\begin{tabular}{|c|c|}
\hline Estudiante & Motivo \\
\hline $\mathbf{A}$ & Para jugar y contestar los mensajes \\
\hline B & Porque tengo que contestar los mensajes \\
\hline $\mathbf{C}$ & $\begin{array}{l}\text { Porque estoy muy aburrida dentro del aula y en las explicaciones } \\
\text { del profe }\end{array}$ \\
\hline $\mathbf{D}$ & $\begin{array}{l}\text { Lo utilizo cuando es importante, además cuando me llama alguien } \\
\text { de mi casa }\end{array}$ \\
\hline $\mathbf{E}$ & Para entretenerme con los juegos y aplicaciones de mi celular \\
\hline $\mathbf{F}$ & $\begin{array}{l}\text { Lo utilizo para hablar, para meterme al facebook y mandar men- } \\
\text { sajes }\end{array}$ \\
\hline G & $\begin{array}{l}\text { Porque a veces es muy aburrida la materia que nos dan o no nos } \\
\text { dan tanta materia }\end{array}$ \\
\hline $\mathbf{H}$ & Porque me mandan mensajes y los tengo que contestar \\
\hline I & Para mandar o contestar mensajes o para usar el internet \\
\hline $\mathbf{J}$ & $\begin{array}{l}\text { Porque a veces la materia es muy aburrida o a veces urge contestar } \\
\text { una llamada }\end{array}$ \\
\hline $\mathbf{K}$ & Porque es muy aburrido poner atención y el celular es más divertido \\
\hline $\mathbf{L}$ & Cuando estoy sin hacer nada y estoy muy aburrido \\
\hline M & Porque a veces tengo mucha pereza y lo saco para mandar mensajes \\
\hline $\mathbf{N}$ & $\begin{array}{l}\text { Porque paso todo el día hablando con mi novio y jugando con el } \\
\text { celular }\end{array}$ \\
\hline $\mathbf{O}$ & $\begin{array}{l}\text { Porque las clases me parecen demasiado aburridas o no hay nada } \\
\text { que hacer }\end{array}$ \\
\hline
\end{tabular}

Fuente: Resultados obtenidos del cuestionario aplicado a los estudiantes de la sección 7-A de un colegio de San José.

Como se puede observar en la Tabla 2, el grupo de estudiantes encuestados utilizan el celular mayoritariamente para comunicarse de manera oral y escrita. Otros de los usos que le dan es utilizar internet, jugar, entre otros. Lo indicado antes constata la presencia de tecnologías celulares y su libre uso dentro del aula de clase. De igual manera, la matriz permite resaltar la opinión de 7 de los encuestados al indicar que la clase es muy aburrida, razón por la cual prefieren utilizar el teléfono celular para entretenerse. 
Tal como lo indica Reyes 2008, puesto que los teléfonos celulares y otras tecnologías están presentes en el aula, una alternativa es usarlos como un recurso didáctico regulado y guiado por el docente, puesto que no se puede dejar de lado que las tecnologías pueden ser de gran ayuda para generar un aprendizaje atractivo, es decir, más allá de su prohibición, lo más apropiado sería generar un uso adecuado de las tecnologías en el aula, en las cuales el docente pueda apoyarse (cuando los temas lo permitan) como un recurso o material didáctico que sirva para facilitar la mediación pedagógica.

Tabla 3. Opinión de los estudiantes del uso de tecnologías y su rendimiento académico

\begin{tabular}{|c|c|}
\hline Estudiante & Opinión \\
\hline $\mathbf{A}$ & $\begin{array}{l}\text { El uso no afecta mi rendimiento académico porque también pongo } \\
\text { atención en las explicaciones. }\end{array}$ \\
\hline B & $\begin{array}{l}\text { Sí afecta el rendimiento al menos en mi caso; yo sé cuando tengo } \\
\text { que guardar mi celular. }\end{array}$ \\
\hline $\mathbf{C}$ & No afecta el rendimiento porque puedo hacer varias cosas a la vez. \\
\hline $\mathbf{D}$ & $\begin{array}{l}\text { No afecta el rendimiento académico porque a mí en lo personal mi } \\
\text { celular no me distrae. }\end{array}$ \\
\hline $\mathbf{E}$ & No afecta porque a mí me va siempre bien \\
\hline $\mathbf{F}$ & Sí afecta mi rendimiento porque me distraen y no pongo atención \\
\hline G & Sí afecta el rendimiento porque distrae al estudiante durante las lecciones. \\
\hline $\mathbf{H}$ & Sí afecta porque no deja que uno aprenda. \\
\hline $\mathbf{I}$ & No afecta porque el profesor sólo escribe en la pizarra. \\
\hline $\mathbf{J}$ & No porque estudio y la tecnología en su debido momento. \\
\hline $\mathbf{K}$ & No afecta el rendimiento porque el que quiere estudia y nada de celulares \\
\hline $\mathbf{L}$ & $\begin{array}{l}\text { Sí afecta porque me cuesta mucha la materia y con teléfono me } \\
\text { distrae mucho. }\end{array}$ \\
\hline $\mathbf{M}$ & Sí afecta, pero a mí no porque no me cuesta la materia del profesor. \\
\hline $\mathbf{N}$ & $\begin{array}{l}\text { Sí afectaría mi rendimiento porque uno tiene que usar el teléfono } \\
\text { en el tiempo libre }\end{array}$ \\
\hline $\mathbf{O}$ & $\begin{array}{l}\text { Sí afecta, aparte de que la clase no es dinámica el uso del celular } \\
\text { nos distrae a todos }\end{array}$ \\
\hline $\mathbf{P}$ & Sí afecta porque es una distracción \\
\hline
\end{tabular}

Nota: Resultados obtenidos del cuestionario aplicado a los estudiantes de la sección 7-A de un colegio de San José. 
Como se puede observar en la Tabla 3, siete de los sujetos cuestionados, dicen que el uso del celular no afecta su rendimiento académico. Al respecto, expresaron tener un buen rendimiento académico y que de todos modos lo que deben hacer es copiar de la pizarra en el desarrollo de las lecciones, es decir, no requieren mayor concentración en las clases de Ciencias. La opinión de estos educandos afirma lo indicado en la Tabla 2 al aducir que las clases son aburridas.

Cabe añadir que lo anterior se pudo constatar en la observación de clase, puesto que lo único que realizaron los estudiantes fue copiar de la pizarra lo que previamente estaba escrito (de clases anteriores), de acuerdo con lo solicitado por el docente.

Por su parte, los restantes nueve estudiantes encuestados indicaron que sí les afecta su rendimiento académico al utilizar dentro del desarrollo de las lecciones el celular, ya que aducen distraerse. En cuanto al rendimiento académico en la asignatura de Ciencias,se pudo comprobar con el registro de calificaciones lo expuesto antes, puesto que de los dieciséis estudiantes del estudio, 9 tenían notas inferiores a 65 en los 2 primeros trimestres del año.

En opinión del docente, el uso de tecnologías celulares dentro del desarrollo de sus clases, sí interfiere en el proceso de aprendizaje de los estudiantes, por ende, el rendimiento académico. Él refirió tener conocimiento de su uso dentro del aula, y tratar de regularlo, indicándoles apagarlos, pero expuso que los educandos no acatan la propuesta. 
Tabla 4. Opinión de los estudiantes del uso de tecnologías por parte del profesor

\begin{tabular}{|c|l|}
\hline Estudiante & \multicolumn{1}{|c|}{ Opinión } \\
\hline A & $\begin{array}{l}\text { Me gustaría que el profesor aplique el uso de las tecnologías } \\
\text { porque haría más interesante la clase. } \\
\text { No me gustaría porque uno tiene que ir a estudiar al colegio y eso } \\
\text { sería una gran distracción. } \\
\text { B }\end{array}$ \\
C & Sí para que el celular tenga una utilidad más importante. \\
D & Sí me gustaría para que no me regañe mucho en clase. \\
E & Sí porque el profesor no molestaría tanto. \\
F & Sí porque las clases serían menos aburridas de lo que son. \\
G & Sí porque así no nos regañan por sacar el celular. \\
H & Sí me gustaría porque las clases serían más interesantes. \\
I & Sí para que la clase sea más entretenida y no aburrida como siempre. \\
J & Sí porque la clase la haría más interesante. \\
K & Me da igual \\
L & No porque los estudiantes se distraerían en clases. \\
M & Sí porque las lecciones serían más interesantes. \\
$\mathbf{N}$ & Sí para que las lecciones se vuelvan más interactivas. \\
$\mathbf{O}$ & Sí me gustaría, para aprender de ellas y hacer la clase bonita \\
P & $\begin{array}{l}\text { Sí porque la clase sería más divertida y los estudiantes pondrían } \\
\text { más atención. }\end{array}$ \\
\hline
\end{tabular}

Fuente: Resultados obtenidos del cuestionario aplicado a los estudiantes de la sección 7-A de un colegio de San José.

Como se puede apreciar en la Tabla 4, la mayoría (13) de los educandos están anuentes a que el docente implemente en las clases tecnologías de manera que sean más atractivas, ya que como se indicó antes las consideran aburridas. Lo anterior lo resalta Galvis (1991) al indicar que la tecnología puede permitir un entorno satisfactorio del aprendizaje de los estudiantes. De manera, que el docente como mediador permita que los estudiantes sean capaces de llegar a construir su propio conocimiento, es decir, que participe de la construcción de su propio conocimiento, utilizando estrategias y materiales acordes con el nivel del educando y congruente con las preferencias de los educandos. En el caso particular, 
se puede sacar provecho de los recursos de comunicación que se tienen a mano en la clase como son los teléfonos y sus diferentes aplicaciones, pues como se muestra en la Tabla 1, la mayoría de los estudiantes del estudio disponen de un celular con diversas aplicaciones.

En cuanto a la entrevista realizada al educador, permitió confirmar varios aspectos, por ejemplo, ratifica la existencia de una marcada influencia en el uso de tecnologías dentro del aula; el celular de manera especial es utilizado con mucha frecuencia, sin ningún fin educativo por parte de los estudiantes, a pesar de que se les pide que no lo utilicen en el desarrollo de las lecciones.

De igual manera, parte de las interrogantes versaron acerca de la importancia del uso del celular como instrumento para integrar los objetivos de aula, a lo cual indicó tener claridad de la importancia, pero no lo usa en clase pues ellos pueden aumentar la distracción y desorden. Si bien los estudiantes poseen un celular, es difícil hacer conciencia en que se va a utilizar como recurso didáctico dentro del aula y sí para jugar y ver otros elementos en internet, por mencionar un ejemplo, sobre todo porque los estudiantes lo ven como una fuente de diversión, unido a que en sétimo es difícil el control de la disciplina por el grado de madurez de los educandos.

La poca anuencia del docente por implementar tecnologías en el desarrollo de las lecciones de Ciencias, se contrasta en la Tabla 4 donde se expone en opinión de los estudiantes que el docente no implementa tecnologías en la clase a pesar de ser del agrado de ellos.

Así mismo indicó, que contar con tecnologías es bueno, ya que reforzaría el nivel de comprensión por parte de los estudiantes, pero que si no se saben utilizar se pueden convertir en un componente negativo, es decir, no se trata de hacer lo mismo utilizando tecnologías, sin embargo indicó nunca haber integrado tecnologías como recurso de mediación en sus clases, lo que confirma que el docente se limita a hacer siempre lo mismo en las lecciones, dejando de lado la diversidad didáctica que debe existir en cualquier aula, para propiciar un aprendizaje acorde con las expectativas y necesidades de los educandos. Este elemento se constata en la Tabla 2, donde los estudiantes dicen no sentirse motivados en las lecciones de ciencias, razón por la cual refieren entretenerse utilizando implementos de comunicación dentro de las lecciones, puesto que no impide copiar de la pizarra, que es lo que generalmente se hace en las lecciones de Ciencas. 
A propósito de lo recién indicado, el profesional indica que los recursos didácticos implementados en el desarrollo de sus lecciones le resultan buenos, por ese motivo siempre pone en práctica, cabe destacar que al referirse a recursos didácticos hace alusión a prácticas, resolver problemas y uso de fotocopias.

Lo expresado por el docente contradice los objetivos de la mediación pedagógica, puesto que lo que importa es el sentimiento que tenga el estudiante hacia la clase. El que dicen no estar a gusto con el desarrollo de las lecciones, razón por la cual prefieren realizar otras actividades como utilizar el celular. Como lo expresa Malagón 2009, citando a Gutiérrez y Prieto 1999 “ (...) la mediación puede surgir del trabajo en el aula y depende casi siempre de la capacidad y la pasión del docente" (s.p).

Propiciar en las aulas una mediación pedagógica en busca de la construcción de mejores conocimientos requiere romper con la idea de que educación es transmisión de conocimientos del docente hacia los educandos, es necesario creer y practicar una educación donde el diálogo, la acción mental, el debate y las experiencias significativas sean prácticas fundamentales a diario. Desde esta perspectiva teórica, el docente asume el papel de guía que propone actividades nuevas que originen conflicto cognitivo en sus estudiantes, por otro lado, el educando debe ser protagonista de su proceso, buscará soluciones a los conflictos, pensará, reflexionará y se formará un criterio propio (Vygotski, 1997).

En cuanto a lo observado en clases, se pudo constastar que no había motivación por parte de los educandos en la clase de Ciencias, lo cual propiciaba su dispersión y poca atención hacia la materia. Por su parte, el docente asumió un rol pasivo, simplemente pidió que ranscribieran lo que estaba escrito previamente en la pizarra y una vez que los estudiantes terminaban de escribir, les indicaba repasar la materia, lo cual se puede atribuir a una falta de planificación de la clase.

Lo anterior, puede ser la justificante de que los estudiantes utilizaran sus aparatos de comunicación constantemente para escuchar música, enviar mensajes, no acatar las pocas instrucciones dadas por el docente y hablar de cualquier tema con tal de pasar el tiempo. Lamentablemente, se observó que el tiempo de esas lecciones fue perdido en su totalidad, tanto por los estudiantes como por el docente, quien se limitó a poner en orden lo que parecía ser su portafolio con materia. 


\section{Conclusiones}

Por medio de los resultados obtenidos y su respectivo análisis, se pueden rescatar las siguientes conclusiones propias de esta investigación y de la muestra estudiada, por lo que no pueden ser generalizadas a otras poblaciones.

- $\quad$ Se identificó que los aparatos electrónicos están presentes dentro el aula de clase. Por su parte, los principales tipos de tecnologías presentes en el desarrollo de las lecciones de Ciencias fueron los celulares.

- Los estudiantes utilizan en clase el celular principalmente para comunicarse de manera escrita.

- $\quad$ Se identificó que los celulares sí influyen en el rendimiento académicos de los educandos en estudio, ya que los distrae, elemento que fue confirmado por varios de los estudiantes y el docente.

- $\quad$ Lo anterior se puede unir a que el docente por lo general utiliza métodos y técnicas en la mediación tradicionales como son copiar de la pizarra y fotocopias. Lo indicado antes influye de manera considerable en que los estudiantes utilicen sus aparatos electrónicos, ya que si la clase no es "provechosa", diversa o interesante, los estudiantes pierden todo interés e incluso pierden hasta la motivación de estar en esa clase, razón por la cual consideran las lecciones de Ciencias poco atractivas.

- La presencia de aparatos tecnológicos en los salones de clase son un hecho, prácticamente cualquier estudiante tiene acceso a ellos lo cual genera que su presencia sea inevitable en las aulas. Las nuevas generaciones evolucionan al ritmo en el que evoluciona la tecnología, por ende, la tecnología debe ser un aliado en el campo educativo si se sabe implementar.

- $\quad$ Las TIC pueden ser incorporadas en el proceso educativo. Pero no se trata de hacer más de lo mismo incorporándolas, puesto que ellas por sí solas no son suficientes. El reto no radica en los recursos, radica en la mediación pedagógica desarrollada en el aula. 


\section{Referencias bibliográficas}

Barrantes, R. (2006). Investigación: un camino al conocimiento un enfoque cualitativo $y$ cuantitativo. Costa Rica, San José: EUNED.

Bessone, N. (2005). El cambio llegará a partir de y en la institución escuela. Recuperado de http://foromediacion.blogspot.com/2008/04/observatorio-mediacin-boletin-bitartoki.html

Cuevas, F. \& Alvarez, V. (2010). Las TIC en la educación secundaria costarricense: Análisis comparativo de estudiantes y docentes.San José, C.R: Coordinación Educativa y Cultural Centroamericana, CECC/PROSIC.

Galvis, A. (1991). Reflexión acerca del uso del computador en Educación primaria y secundaria. Revista informática Educativa, 1(4),11-38. Recuperado de http://www. colombiaaprende.edu.co/html/mediateca/1607/articles-127396_archivo.pdf

Galvis, A. (2004, Junio). Oportunidades de las Tecnologías de Información y Comunicación. Recuperado de http://www.karisma.org.co/documentos/softwareredp/ tic-galvis-articles 73523_archivo.pdf

González, E., Leal, F. \& Romero, G. (2011). Perfil socioafectivo y socioeconómico de los estudiantes del III ciclo de la educación general básica que habitan en la comunidad de La Guaria y asistieron al colegio técnico profesional de Puerto Viejo de Sarapiqui de Heredia. [Tesis de Licenciatura sin publicar]. Costa Rica: Universidad Nacional.

Gurdián, A. (2007). El paradigma cualitativo en la investigación socio-educativa. Costa Rica, San José: CECC, AECI.

Gutiérrez Pérez, F. \& Prieto Castillo, D. (2004). Mediación pedagógica. (10 ed.). Proyecto de desarrollo Santiago-PRODESSA. Santiago, Guatemala: Ediciones la Copia Fiel.

Hernández, R., Fernández, C., \& Baptista, P. (2006). Metodología de la investigación. (4 ed). México, México DF: McGrawHill.

Leitón, K. (2010). Análisis de los factores afectivos y cognitivos que inciden en el rendimiento académico, en la asignatura de matemática, en la modalidad nocturno académico: estudio de caso en la sección 9-3 del colegio nocturno de Guácimo, durante el curso lectivo 2008. [Tesis de licenciatura sin publicar]. Costa Rica: Universidad Nacional.

Malagón, J. (2009). La mediación pedagógica. Recuperado de http://www.ucm.es/info/ mediars/Biblioteca/files/d04c3f97c41bf19be649f41e63de3c0b-2.html

Matarrita, L., Serrano, J., Torres, I. \& León, G. (2012). Factibilidad de la utilización de recursos tecnológicos en la implementación del eje transversal cultura ambiental para el desarrollo sostenible en la Educación General Básica de Costa Rica. Revista Electrónica Educare, 16(3), 127-143. Recuperado de http://www. revistas.una.ac.cr/index.php/EDUCARE/issue/current

Olier, K. (s. f.). Efectos de un programa de educación virtual sobre los conocimientos de los docentes acerca el uso de las nuevas tecnologías de la información y comunicación con fines educativos. Tesis de Licenciatura.Barranquilla: Universidad del Norte. Recuperado de http://www.udenar.edu.co/viceacademica/EDUCACION\%20EN\%20ESPACIOS\%20VIRTUALES/EFECTOS\%20DE\%20 UN\%20PROGRAMA\%20DE\%20EDUCACION\%20 VIRTUAL.doc 
Pérez, R.A. (2009). El constructivismo en los espacios educativos. San José, C.R: Coordinación Educativa y Cultural Centroamericana, CECC/SICA.

Piscitelli, A. (s. f.).Inmigrantes digitales vs nativos digitales. Recuperado http://50.56.237.235/mediasite/sites/default/files/19_Nativos-Digitales.pdf

Reyes, F. (2008). La era digital: valor y uso de las nuevas tecnologías educativas. Revista digital Universitaria, 2(8), 1-7. Recuperado de http://www.revista.unam. $\mathrm{mx} / \mathrm{vol} .9 /$ num2/art08/feb_art08.pdf

Sánchez, M.L. (2011). El uso del celular para desarrollar el pensamiento crítico, reflexivo y analítico.Etic@net,9,(11),196-212. Recuperado de http://www.ugr. es/ sevimeco/revistaeticanet/numero11/Articulos/Formato/articulo9.pdf

Vygotski, L. S. (1997). Obras escogidas (Tomo V). Madrid: Aprendizaje Visor. 